\title{
Extracting Information on Flow Direction in Multivariate Time Series
}

\author{
Chunfeng Yang, Régine Le Bouquin Jeannès, Gérard Faucon, and Huazhong Shu, Senior Member, IEEE
}

\begin{abstract}
Phase slope index is a measure which aims at detecting causal relation of interdependence in multivariate time series. One drawback of this approach relies in its incapability to distinguish the direct and indirect relations. So, in order to identify only direct relations, we propose to replace the ordinary coherence function used in the phase slope index with the partial coherence. Furthermore, we consider and compare two estimators of the coherence functions, the first one based on Fourier transform and the second one on an autoregressive model. In order to cope with the difficult issue of bidirectional flow, which cannot be addressed by the coherence based phase slope index, we propose another index based on the directed transfer function. Experimental results support the relevance of the new indices, both based on autoregressive modeling, in multivariate time series.
\end{abstract}

Index Terms-Directed transfer function, ordinary coherence, partial coherence, phase slope index.

\section{INTRODUCTION}

I $\mathrm{N}$ neuroscience, understanding of brain functioning requires the investigation of activated cortical networks, in particular the detection of interactions between different cortical sites. Over the last decade, some measures have been investigated to deal with functional and effective connectivity in multiple areas, such as in chaotic systems, and multivariate neurobiological signals [1]-[5]. Recently, a measure named Phase Slope Index (PSI) was proposed by Nolte [6] to detect information flow direction. This method, based on linear phase between two signals, estimates the causal direction by computing the slope of the phase of ordinary coherence (OC) function. However, in multivariate time series, when two time series display direct and/or indirect causal relations, PSI based on ordinary coherence function fails to distinguish them. The intended application concerns the analysis of electroencephalographic signals recorded during epileptic seizures in patients with drug-resistant epilepsy, and, in this context, different phases are observed during the seizure process, including a fast onset activity (FOA) [7]. The goal is to determine how cerebral

C. Yang, R. Le Bouquin Jeannès, and G. Faucon are with the INSERM, U642, 35000 Rennes, France, the Université de Rennes 1, LTSI, 35000 Rennes, France, and also with the Centre de Recherche en Information Biomédicale Sino-Français (CRIBs), 35000 Rennes, France (e-mail: chunfeng.yang@univrennes1.fr; regine.le-bouquin-jeannes@univ-rennes1.fr; faucon.gerard@gmail. com).

H. Shu is with Laboratory of Image Science and Technology, School of Computer Science and Engineering, Southeast University, 210096 Nanjing, China and also with the Centre de Recherche en Information Biomédicale Sino-Français (CRIBs), Nanjing, China (e-mail: shu.list@seu.edu.cn). structures get involved during this FOA, in particular, in which way some structure can "drive" other ones. In order to detect direct causal relations and distinguish patterns of connectivity, we propose a new phase slope index based on partial coherence (PC) function instead of ordinary coherence function. If ordinary coherence function can be obtained using Fourier transform as in [6], another approach consists in computing ordinary and partial coherences using autoregressive (AR) modeling of signals as proposed hereafter. In a second step, to face with the symmetric character of the coherence function and be able to detect bidirectional relations between signals, we introduce another phase slope index based on directed transfer function (DTF) [8] instead of ordinary coherence function.

\section{METHODS}

PSI is a method to evaluate the information flow direction in multivariate time series [6]. After recalling the principle of this method, we derive the expression of a new PSI based on the partial coherence function, and then the expression of another new index based on the DTF.

\section{A. PSI Principle}

The basic hypothesis relies on the phase linearity between signals. PSI is based on the slope of the phase of the crossspectrum between two time series $x_{m}(t)$ and $x_{n}(t)$. The idea is to define an average measure in such a way that this quantity properly represents relative time delays between signals. This quantity, noted $\mathrm{PSI}_{m n}$, is defined by

$$
\operatorname{PSI}_{m n}=\operatorname{Im}\left(\sum_{f \in F} C_{m n}^{*}(f) C_{m n}(f+\delta f)\right)
$$

where $C_{m n}(f)$ is the coherence function between signals $x_{m}$ and $x_{n}, \delta f$ is the frequency resolution, $\operatorname{Im}(\bullet)$ denotes the imaginary part and the asterisk denotes conjugate value. $F$ is the set of frequencies over which the index is computed. In this equation, the coherence function used by Nolte in [6] is the ordinary coherence between signals $x_{m}(t)$ and $x_{n}(t)$, noted as $O C_{m n}(f)$ hereafter, and defined as follows:

$$
O C_{m n}(f)=\frac{S_{m n}(f)}{\sqrt{S_{m m}(f) S_{n n}(f)}}
$$

where $S_{m m}(f)$ and $S_{n n}(f)$ are the auto-spectral density functions of signals $x_{m}(t)$ and $x_{n}(t)$ respectively, and $S_{m n}(f)$ is the cross-spectral density function. They are given by

$$
S_{m n}(f)=E\left[X_{m}(f) X_{n}^{*}(f)\right]
$$

where $X_{m}(f)$ is the Fourier transform of the signal $x_{m}(t)$ and $E[\bullet]$ denotes the expectation. The magnitudes of the coherences allow weighting the phase difference between two consecutive frequencies and, consequently, decrease its impact when they 
are low. The sign of PSI indicates the flow direction and its magnitude increases along with the delay. Given (1)-(3), when the information flow is from $x_{m}(t)$ to $x_{n}(t), \mathrm{PSI}_{m n}$ is positive. In the following, PSI using the OC is named PSI-OC:

$$
\mathrm{PSI}_{m n}-\mathrm{OC}=\operatorname{Im}\left(\sum_{f \in F} O C_{m n}^{*}(f) O C_{m n}(f+\delta f)\right) .
$$

Given (3), the auto-spectral and cross-spectral density functions may be obtained by two different techniques, either from direct Fourier transforms of signals $x_{m}(t)$ and $x_{n}(t)$ as in [6], or from AR modeling. In the first one, the expectation required to get the spectral density functions is obtained by averaging and overlap. In the second one, the methodology in the multivariate case can be derived as follows. Let $x_{1}, x_{2}, \ldots, x_{Q}$ be $Q$ zero-mean signals whose discrete-time observations are noted $x_{1}(t), x_{2}(t), \ldots, x_{Q}(t), t=1,2, \ldots, T$, where $T$ is the signal length. If we model the observations by a multivariate AR model of order $p$, we write

$$
\left(\begin{array}{c}
x_{1}(t) \\
\vdots \\
x_{Q}(t)
\end{array}\right)=\sum_{k=1}^{p} A_{k}\left(\begin{array}{c}
x_{1}(t-k) \\
\vdots \\
x_{Q}(t-k)
\end{array}\right)+\left(\begin{array}{c}
w_{1}(t) \\
\vdots \\
w_{Q}(t)
\end{array}\right)
$$

where each signal depends not only on its own past but also on the past of the other signals [5]. $w_{m}(t), m=1,2, \ldots, Q$, are white Gaussian noises, and

$$
A_{k}=\left(\begin{array}{ccccc}
\alpha_{11}(k) & \alpha_{12}(k) & \ldots & \ldots & \alpha_{1 Q}(k) \\
\vdots & \vdots & \vdots & \vdots & \vdots \\
\vdots & \vdots & \vdots & \alpha_{m n}(k) & \vdots \\
\vdots & \vdots & \vdots & \vdots & \vdots \\
\alpha_{Q 1}(k) & \cdots & \cdots & \cdots & \alpha_{Q Q}(k)
\end{array}\right) .
$$

The coefficient $\alpha_{m n}(k)$ evaluates the linear interaction of $x_{n}(t-k)$ on $x_{m}(t)$, whatever $m, n$. These coefficients are estimated by least squares method [9]. Using the lag operator $L\left(L x_{m}(t)=x_{m}(t-1), m=1,2, \ldots, Q\right)$, we rewrite (5) in the following form:

$$
L_{-} A_{k}\left(\begin{array}{c}
x_{1}(t) \\
\vdots \\
x_{Q}(t)
\end{array}\right)=\left(\begin{array}{c}
w_{1}(t) \\
\vdots \\
w_{Q}(t)
\end{array}\right)
$$

where (see the equation at the bottom of the page).
Applying the Fourier transform to both sides of (7) leads to

$$
\begin{aligned}
\underbrace{\left(\begin{array}{cccc}
D_{11}(f) & D_{12}(f) & \cdots & D_{1 Q}(f) \\
D_{21}(f) & D_{22}(f) & \cdots & D_{2 Q}(f) \\
\vdots & \vdots & \ddots & \vdots \\
D_{Q 1}(f) & D_{Q 2}(f) & \cdots & D_{Q Q}(f)
\end{array}\right)}_{D(f)} & \left(\begin{array}{c}
X_{1}(f) \\
\vdots \\
X_{Q}(f)
\end{array}\right) \\
& =\left(\begin{array}{c}
W_{1}(f) \\
\vdots \\
W_{Q}(f)
\end{array}\right)
\end{aligned}
$$

where the components of the coefficient matrix $D(f)$ are $D_{m n}(f)=\left\{\begin{array}{l}1-\sum_{k=1}^{p} \alpha_{m n}(k) e^{-2 i \pi f k}, m=n \\ -\sum_{k=1}^{p} \alpha_{m n}(k) e^{-2 i \pi f k}, m \neq n\end{array}\right.$

Defining the transfer function $H(f)$ as the inverse of the coefficient matrix $D(f)$, we obtain

$$
\left(\begin{array}{c}
X_{1}(f) \\
\vdots \\
X_{Q}(f)
\end{array}\right)=\underbrace{\left(\begin{array}{cccc}
H_{11}(f) & H_{12}(f) & \cdots & H_{1 Q}(f) \\
H_{21}(f) & H_{22}(f) & \cdots & H_{2 Q}(f) \\
\vdots & \vdots & \ddots & \vdots \\
H_{Q 1}(f) & H_{Q 2}(f) & \cdots & H_{Q Q}(f)
\end{array}\right)}_{H(f)}\left(\begin{array}{c}
W_{1}(f) \\
\vdots \\
W_{Q}(f)
\end{array}\right) .
$$

Then, we get the spectral density matrix

$$
\begin{aligned}
S(f) & =\left(\begin{array}{cccc}
S_{11}(f) & S_{12}(f) & \cdots & S_{1 Q}(f) \\
S_{21}(f) & S_{22}(f) & \cdots & S_{2 Q}(f) \\
\vdots & \vdots & \ddots & \vdots \\
S_{Q 1}(f) & S_{Q 2}(f) & \cdots & S_{Q Q}(f)
\end{array}\right) \\
& =H(f) \Gamma H^{\dagger}(f)
\end{aligned}
$$

where

$\Gamma=\left(\begin{array}{cccc}\operatorname{var}\left(w_{1}\right) & \operatorname{cov}\left(w_{1}, w_{2}\right) & \cdots & \operatorname{cov}\left(w_{1}, w_{Q}\right) \\ \operatorname{cov}\left(w_{2}, w_{1}\right) & \operatorname{var}\left(w_{2}\right) & \cdots & \operatorname{cov}\left(w_{2}, w_{Q}\right) \\ \vdots & \vdots & \ddots & \vdots \\ \operatorname{cov}\left(w_{Q}, w_{1}\right) & \operatorname{cov}\left(w_{Q}, w_{2}\right) & \cdots & \operatorname{var}\left(w_{Q}\right)\end{array}\right)$, $\operatorname{cov}(\cdot)$ stands for covariance [5], and the symbol $\dagger$ denotes Hermitian transpose. Finally, the corresponding PSI-OC can be calculated using (1), (2), and (10).

\section{B. PSI Based on Partial Coherence (PC)}

The partial coherence function gives the level of coupling between two signals $x_{m}(t)$ and $x_{n}(t)$ when the influence of the $Q-2$ other signals is removed [10]. It is defined by

$$
P C_{m n \cdot X_{Q-2}}(f) \triangleq \frac{S_{m n \cdot X_{Q-2}}(f)}{\sqrt{S_{m m \cdot X_{Q-2}}(f) S_{n n \cdot X_{Q-2}}(f)}}
$$

$$
L_{-} A_{k}=\left(\begin{array}{cccc}
1-\sum_{k=1}^{p} \alpha_{11}(k) L^{k} & -\sum_{k=1}^{p} \alpha_{12}(k) L^{k} & \cdots & -\sum_{k=1}^{p} \alpha_{1 Q}(k) L^{k} \\
-\sum_{k=1}^{p} \alpha_{21}(k) L^{k} & 1-\sum_{k=1}^{p} \alpha_{22}(k) L^{k} & \cdots & -\sum_{k=1}^{p} \alpha_{2 Q}(k) L^{k} \\
\vdots & \vdots & \ddots & \vdots \\
-\sum_{k=1}^{p} \alpha_{Q 1}(k) L^{k} & -\sum_{k=1}^{p} \alpha_{Q 2}(k) L^{k} & \cdots & 1-\sum_{k=1}^{p} \alpha_{Q Q}(k) L^{k}
\end{array}\right)
$$


where $X_{Q-2} \triangleq x_{1} \cdots x_{m-1} x_{m+1} \cdots x_{n-1} x_{n+1} \cdots x_{Q}$. $S_{m n \cdot X_{Q-2}}(f)$ is the conditioned cross-spectral density function between signals $x_{m}(t)$ and $x_{n}(t)$ given $X_{Q-2} \cdot S_{m m \cdot X_{Q-2}}(f)$ and $S_{n n \cdot X_{Q-2}}(f)$ are conditioned auto-spectral density functions of signals $x_{m}(t)$ and $x_{n}(t)$ respectively. In [10], Bendat and Piersol gave the methodology to obtain $S_{m n \cdot X_{Q-2}}(f)$, $S_{m m \cdot X_{Q-2}}(f)$, and $S_{n n \cdot X_{Q-2}}(f)$. This methodology is introduced simply in the following for $m=1$ and $n=2$. Rewriting (10) in the following form

$$
\left.\begin{array}{rc|cccc}
S_{11}(f) & S_{12}(f) & S_{13}(f) & S_{14}(f) & \cdots & S_{1 Q}(f) \\
S_{21}(f) & S_{22}(f) & S_{23}(f) & S_{24}(f) & \cdots & S_{2 Q}(f) \\
\hline S_{31}(f) & S_{32}(f) & S_{33}(f) & S_{34}(f) & \cdots & S_{3 Q}(f) \\
\vdots & \vdots & \vdots & \vdots & \ddots & \vdots \\
S_{Q 1}(f) & S_{Q 2}(f) & S_{Q 3}(f) & S_{Q 4}(f) & \cdots & S_{Q Q}(f)
\end{array}\right)
$$

the conditioned spectral matrix between signals $x_{1}(t)$ and $x_{2}(t)$ given $X_{Q-2}$ is defined by

$$
\left(\begin{array}{cc}
S_{11 \cdot X_{Q-2}}(f) & S_{12 \cdot X_{Q-2}}(f) \\
S_{21 \cdot X_{Q-2}}(f) & S_{22 \cdot X_{Q-2}}(f)
\end{array}\right)=B_{1}-B_{2} B_{4}^{-1} B_{3} .
$$

In PSI given in (1), we replace the coherence function with the partial coherence and the corresponding PSI is noted PSI-PC: the influence of the $Q-2$ other signals is removed and only the direct influence between $x_{m}(t)$ and $x_{n}(t)$ is considered:

$\mathrm{PSI}_{m n}-\mathrm{PC}=\operatorname{Im}\left(\sum_{f \in F} P C_{m n \cdot X_{Q-2}}^{*}(f) P C_{m n \cdot X_{Q-2}}(f+\delta f)\right)$.

\section{PSI Based on Directed Transfer Function (DTF)}

Directed transfer function was proposed by Kaminski and Blinowska in [8]. From (9), the definition of DTF from $x_{m}(t)$ to $x_{n}(t)$ is defined by

$$
D T F_{m n}(f) \triangleq \frac{H_{n m}(f)}{\sqrt{\sum_{n=1}^{Q}\left|H_{n m}(f)\right|^{2}}}
$$

where $H_{n m}(f)$ is the $(n, m)$ element of the matrix $H(f)$, corresponding to the contribution of signal $x_{m}(t)$ onto signal $x_{n}(t)$. $D T F_{m n}$ is a multichannel causality measure based on the AR model and measures the flow from channel $m$ to channel $n$. It is constructed using elements of the transfer matrix of the AR model. This matrix is not symmetrical and, the value of the DTF, at a given frequency, represents a ratio between the inflow to channel $n$ from channel $m$ to all the inflows to channel $n$. So, contrary to ordinary coherence, it can deal with bidirectional flow and answer to the question of effective connectivity. DTF is able to quantify the strength of such connectivity but the supplementary interest of the PSI based indicator relies on the phase information carried by each element of the transfer function used in the DTF. The relevance of PSI-DTF consists in using on the one hand the amplitude of the directed transfer function and on the other hand the variation of its phase between two adjacent frequencies. So, averaging over the frequency band allows assessing not only the strength of the coupling but also the importance of the delay. In the same way as previously, we define a phase slope index based on DTF, noted PSI-DTF, as follows:

$$
\mathrm{PSI}_{m n}-\mathrm{DTF}=\operatorname{Im}\left(\sum_{f \in F} D T F_{m n}^{*}(f) D T F_{m n}(f+\delta f)\right) .
$$

\section{RESULTS}

We tested our approaches on models simulating practical situations. In our simulations, the model order is given by Akaike's criterion [5].

\section{A. First Model}

For the first linear stochastic system we considered, the three following signals were generated:

$$
\left\{\begin{array}{l}
x_{1}(t)=0.95 \sqrt{2} x_{1}(t-1)-0.9025 x_{1}(t-2)+w_{1}(t) \\
x_{2}(t)=-0.5 x_{1}(t-1)+w_{2}(t) \\
x_{3}(t)=0.8 x_{2}(t-2)+c x_{1}(t-4)+w_{3}(t)
\end{array}\right.
$$

where $w_{j}(t), j=1,2,3$, were independent white Gaussian noises with zero means and unit variances; the parameter $c$ was introduced to consider two patterns of causal interactions, either direct $(c=0.5)$ or indirect $(c=0)$ relationships. The sampling frequency was $200 \mathrm{~Hz}$.

In Section II, ordinary and partial coherence functions are used to obtain two phase slope indices, respectively PSI-OC and PSI-PC. As indicated above, spectra can be obtained either by Fourier transform or using multivariate AR modeling. In the following, we denote by PSI-OC(FFT) (resp. PSI-PC(FFT)) the case where the ordinary (resp. partial) coherence is estimated by fast Fourier transform. In the same way, PSI-OC(AR) (resp. PSI-PC(AR)) denotes the case where the ordinary (resp. partial) coherence is estimated by AR modeling.

When using FFT, spectra were obtained using a sliding window of 64-point length and a 50\% overlap. As for AR modeling, it was realized on the whole signal length. Simulation was carried out 100 times on 1024-point signals; the means and standard deviations (sd) were computed and reported in Table I. From this table, two main results can be drawn: i) both PSI-OC and PSI-PC perfectly point out the flow direction of information among the three signals. However, PSI-PC allows distinguishing the direct and indirect relations whatever the computation mode (FFT or AR). For example, in the case $x_{1} \rightarrow x_{3}$, when $c=0$, corresponding to indirect relation, $\mathrm{PSI}_{13}-\mathrm{PC}$ is close to zero, and when $c=0.5$, corresponding to direct relation, $\mathrm{PSI}_{13}-\mathrm{PC}$ increases significantly, whereas $\mathrm{PSI}_{13}$-OC systematically presents important values whatever $c(c=0$ or $c=0.5)$; ii) the results obtained with AR modeling are preferred for two reasons: when there is some direct relation between two signals, the mean values are generally higher with AR modeling and the corresponding sd are lower.

The robustness of these methods against additive disturbing independent noises has also been tested. Choosing signal-tonoise ratios in the range $[5 \mathrm{~dB} ; 10 \mathrm{~dB}]$ did not affect the performance of the indicators and the same conclusions hold.

We also considered the case of nonlinearities as that described in [11] in this context of unidirectional flow. In this situation, it came out that the proposed indicators remained robust when 
TABLE I

Results on PSI-OC AND PSI-PC. The First VAlue IS The MEAN, THE VAlue IN PaRentheses IS THE SD

\begin{tabular}{|c|c|c|c|c|c|c|c|c|}
\hline & \multicolumn{4}{|c|}{ PSI-OC } & \multicolumn{4}{|c|}{ PSI-PC } \\
\hline & \multicolumn{2}{|c|}{$c=0$} & \multicolumn{2}{|c|}{$c=0.5$} & \multicolumn{2}{|c|}{$c=0$} & \multicolumn{2}{|c|}{$c=0.5$} \\
\hline & FFT & AR & FFT & AR & FFT & AR & FFT & AR \\
\hline \multirow{2}{*}{$\mathrm{PSI}_{12}$} & 0.9242 & 0.9545 & 0.9242 & 0.9624 & 0.6198 & 0.5846 & 1.2738 & 1.3419 \\
\hline & $(0.1315)$ & $(0.0770)$ & $(0.1315)$ & $(0.0796)$ & $(0.1423)$ & $(0.0574)$ & $(0.1500)$ & $(0.0899)$ \\
\hline \multirow{2}{*}{$\mathrm{PSI}_{13}$} & 1.7860 & 1.9548 & 2.1132 & 2.4053 & 0.0292 & 0.0076 & 2.0475 & 2.4082 \\
\hline & $(0.2171)$ & $(0,1348)$ & $(0.2855)$ & $(0.2483)$ & $(0.1448)$ & $(0.0092)$ & $(0.3000)$ & $(0.2608)$ \\
\hline \multirow{2}{*}{$\mathrm{PS}_{23}$} & 3.1413 & 3.2289 & 2.7487 & 2.9330 & 2.3932 & 2.4274 & 2.2858 & 2.4288 \\
\hline & $(0.1501)$ & $(0.1241)$ & (0.1881) & $(0.1658)$ & $(0.1730)$ & $(0.1455)$ & $(0.1768)$ & $(0.1547)$ \\
\hline
\end{tabular}

TABLE II

Results on PSI-OC AND PSI-PC. The First VAlue IS the MeAN, THE VALUE IN PARENTHESES IS THE SD

\begin{tabular}{l|c|c}
\hline & PSI-OC & PSI-PC \\
\hline PSI $_{12}$ & 2.4070 & 1.3408 \\
& $(0.2062)$ & $(0.0798)$ \\
& 2.7573 & 0.9889 \\
PSI $_{13}$ & $(0.2526)$ & $(0.2049)$ \\
& -0.7573 & -0.7411 \\
PSI $_{23}$ & $(0.2450)$ & $(0.2587)$ \\
\hline
\end{tabular}

considering the particular case of nonlinear signals with linear coupling.

\section{B. Second Model}

For the second linear stochastic system, the three following signals were generated:

$$
\left\{\begin{array}{l}
x_{1}(t)=0.95 \sqrt{2} x_{1}(t-1)-0.9025 x_{1}(t-2)+w_{1}(t) \\
x_{2}(t)=-0.5 x_{1}(t-1)-0.8 x_{3}(t-3)+w_{2}(t) \\
x_{3}(t)=0.8 x_{2}(t-2)+0.5 x_{1}(t-4)+w_{3}(t)
\end{array}\right.
$$

where $w_{j}(t), j=1,2,3$, were independent white Gaussian noises with zero means and unit variances. In this situation, bidirectional causalities existed between signals $x_{2}$ and $x_{3}$. The sampling frequency was $200 \mathrm{~Hz}$.

\section{Results on PSI-OC and PSI-PC}

Since we already found that the indices based on AR modeling outperform those based on FFT, we only present results on AR modeling. Simulations were carried out 500 times on 1024-point signals, the means and sd were derived and reported in Table II. From this table, we can see that both PSI-OC and PSI-PC can point out the correct information flow when there is only unidirectional causality, e.g., between $x_{1}$ and $x_{2}$. On the other hand, both PSI-OC and PSI-PC fail when bidirectional causality exists, e.g., between $x_{2}$ and $x_{3}$.

\section{Results on PSI-DTF}

Simulation was also carried out 500 times on 1024-point signals, the means and sd were derived and reported in Table III. When there is no information flow, e.g., from $x_{2}$ to $x_{1}$, or from $x_{3}$ to $x_{1}, \mathrm{PSI}_{21}-\mathrm{DTF}$ and $\mathrm{PSI}_{31}-\mathrm{DTF}$ remain close to zero. When some information flow exists, e.g., from $x_{1}$ to $x_{2}$, or from $x_{1}$ to $x_{3}$ in the case of unidirectional flows, or from $x_{2}$ to $x_{3}$ but also from $x_{3}$ to $x_{2}$ in the case of bidirectional flow, PSI-DTF is able to identify the different propagation graphs. For the bidirectional case, the difference obtained between $\mathrm{PSI}_{23}-\mathrm{DTF}$ and $\mathrm{PSI}_{32}$-DTF can be explained by the values of the delays used to generate signals $x_{2}$ and $x_{3}$. Considering a symmetric interaction between two of these signals, $x_{m}$ and $x_{n}$, the respective
TABLE III

Results on PSI-DTF. The First Value is the Mean, THE VALUE IN PARENTHESES IS THE SD

\begin{tabular}{c|ccc}
\hline$x_{m} \rightarrow x_{n}$ & $m=1$ & $m=2$ & $m=3$ \\
\hline$n=1$ & 0 & 0.0456 & 0.0498 \\
& & $(0.0267)$ & $(0.0283)$ \\
$n=2$ & 4.2191 & & 2.7103 \\
& $(0.1988)$ & 0 & $(0.1039)$ \\
$n=3$ & 4.5558 & 1.8231 & \\
& $(0.1981)$ & $(0.0793)$ & 0 \\
\hline
\end{tabular}

values of the PSI-DTF indicator from signal $x_{m}$ to signal $x_{n}$ and from signal $x_{n}$ to signal $x_{m}$ would be comparable. So, PSI-DTF appears as a relevant indicator to detect unidirectional and bidirectional causalities.

\section{CONCLUSION}

In this letter, we focused on information propagation between multi-site observations using a phase slope index based approach. The interest of the technique we proposed can be summarized as follows: i) the introduction of partial coherence instead of ordinary coherence allows dealing with direct causal relations; ii) AR modeling reduces estimator variance. Combining both improvements allows us to distinguish direct and indirect causal relations in multivariable time series with the lowest error; iii) the introduction of directed transfer function instead of ordinary coherence reveals pertinent to deal with bidirectional causal relations. In a future work, we plan to test these approaches to reveal the underlying network organization of an epileptic seizure, in some difficult situation where time shifts between signals strongly vary in time.

\section{REFERENCES}

[1] B. Veeramani, K. Narayanan, A. Prasad, L. D. Iasemidis, A. S. Spanias, and K. Tsakalis, "Measuring the direction and the strength of coupling in nonlinear systems-a modeling approach in the state space," IEEE Signal Process. Lett., vol. 11, pp. 617-620, 2004.

[2] G. C. Carter, "Coherence and time delay estimation," Proc. IEEE, vol. 75, pp. 236-255, 2005.

[3] S. Sabesan, L. Good, K. Tsakalis, A. Spanias, D. Treiman, and L. Iasemidis, "Information flow and application to epileptogenic focus localization from intracranial EEG," IEEE Trans. Neural Syst. Rehab. Eng., vol. 17, pp. 244-253, Jun. 2009.

[4] C. W. J. Granger, "Investigating causal relations by econometric models and cross-spectral methods," Econometrica, vol. 37, pp. 424-438, Aug. 1969.

[5] M. Ding, Y. Chen, and S. L. Bressler, "Granger causality: Basic theory and application to neuroscience," in Handbook of Time Series Analysis, M. Winterhalder, B. Schelter, and J. Timmer, Eds. Hoboken, NJ: Wiley-VCH, 2006, pp. 437-460.

[6] G. Nolte, A. Ziehe, V. V. Nikulin, A. Schlögl, N. Krämer, T. Brismar, and K. R. Müller, "Robustly estimating the flow direction of information in complex physical systems," Phys. Rev. Lett., vol. 100, pp. 234101:1-234101:4, Jun. 2008.

[7] F. Wendling, A. Hernandez, J. J. Bellanger, P. Chauvel, and F. Bartolomei, "Interictal to ictal transition in human temporal lobe epilepsy: Insights from a computational model of intracerebral EEG," J. Clin. Neurophys., vol. 22, pp. 343-356, 2005.

[8] M. J. Kaminski and K. J. Blinowska, "A new method of the description of the information flow in the brain structures," Biol. Cybern., vol. 65, pp. 203-210, 1991.

[9] L. W. P. Biscainho, "AR model estimation from quantized signals," IEEE Signal Process. Lett., vol. 11, pp. 183-185, 2004.

[10] J. S. Bendat and A. G. Piersol, Random Data-Analysis and Measurement Procedures, 2nd ed. New York: Wiley, 1986.

[11] Y. Chen, G. Rangarajan, J. Feng, and M. Ding, "Analyzing multiple nonlinear time series with extended Granger causality," Phys. Lett. A vol. 324, pp. 26-35, 2004. 